\title{
Comunicación, lenguaje y educación: una mirada desde las teorías de la complejidad
}

\section{Communication, language and education: a look from the theories of complexity}

\author{
Rita Flórez Romero 1 \\ Jaime Castro Martínez ${ }^{2}$ \\ Nicolás Arias Velandia 3
}

\section{Resumen}

Este trabajo presenta un modelo conceptual de la relación comunicación-educación desde los planteamientos de la complejidad, utilizando como unidad de análisis la interacción comunicativa. En el nacimiento de las explicaciones desde la complejidad, se explica el concepto de sistema, entendido como un conjunto de elementos y de relaciones entre éstos, que tienden a mantenerse organizadas y de las cuales surgen elementos no preexistentes. De acá se derivan los principales conceptos básicos de teorías de la complejidad para explicar la interacción comunicativa: la autoorganización, la no linealidad y la relación del sistema con otros sistemas subordinados y superordenados. Dichos conceptos explican tres aspectos de la interacción comunicativa en educación: estructuras de conocimiento, relación aprendiz-maestro y ambientes o espacios de conocimiento. Los avances en el marco de relación entre complejidad, educación y comunicación muestran la necesidad de una perspectiva evolutiva integradora de las dinámicas causales de la interacción comunicativa para explicar la complejidad de la realidad educativa. Este nuevo lenguaje debe centrarse en una nueva síntesis de las ciencias naturales y las ciencias sociales.

\section{Palabras clave}

Complejidad, autoorganización, interacción comunicativa, no linealidad, entreveramiento, aprendizaje.

\section{Abstract}

This work presents a conceptual model on the relationship communication - education from the perspective of complexity theories, using communicative interaction as analysis unit. In the birth of complexity explanations, the concept of system is explained, and is understood as a set of elements and relationships among them that tend to stay organized and to generate new elements. From this point of view, the basic concepts in complexity theories-self-organization, non-linearity, and the relationships among the system and other subordinate and superordinate systems -emerge as an explanation to communicative interaction. These concepts explain three communicative interaction aspects in education: knowledge structures, teacher-student relationship and knowledge environments. The new findings on the framework given by the relationship complexity-education-communication shows that a perspective on development and integration in causal dynamics about communicative interaction is needed to explain the complexity inherent to educative reality. This new language should focus on a natural science-social science new synthesis.

\section{Palabras clave}

Estudiante-profesor, práctica docente, lenguas, educación.

Artículo recibido el 18 de junio de 2009 y aprobado el 16 de octubre de 2009.

1 Profesora asociada del Departamento de Comunicación Humana, Facultad de Medicina, Universidad Nacional de Colombia. Correo electrónico: rflorezr@unal.edu.co

2 Profesor asociado a la Facultad de Ciencias Sociales, Politécnico Grancolombiano. Correo electrónico: jcastrom@poligran.edu.co

3 Asistentes de investigación Departamento de Comunicación Humana, Facultad de Medicina. Universidad Nacional de Colombia. Correo electrónico: nariasv@unal.edu.co 
En las últimas décadas, las diversas teorías de la complejidad han permeado de forma importante el campo de las ciencias sociales y la educación. Sin embargo, estas inclusiones se han circunscrito mayoritariamente al trabajo del filósofo y sociólogo Edgar Morin, en lo que se ha llamado pensamiento en complejidad. Los autores de este artículo presentamos una visión complementaria al pensamiento complejo, la cual se basa en pensar el desarrollo de estructuras de conocimiento complejas como procesos autoorganizativos que devienen de la dinámica interactiva de los aprendices con los maestros por medio de principios que exhiben al lenguaje y la comunicación como elementos importantes en la comprensión compleja de los ambientes educativos, de las estructuras cognitivas y de las interacciones en estos ambientes. Sustentamos este aporte a la complejidad por medio de los llamados espacios de conocimiento, entendido como ecosistemas donde el elemento a resaltar es la interacción comunicativa. Para ello, introducimos al lector en diversos conceptos importantes de las teorías complejas, como son: emergencia, autoorganización y no linealidad, y los relacionamos con las dinámicas que se entretejen en los actos y conocimientos de la educación.

Esta aproximación subraya la necesidad de desarrollar una nueva ciencia de la educación sustentada en un enfoque de síntesis, a la vez que sistémico, que supere o complemente la propuesta de la ciencia tradicional analítica. Vemos la necesidad de trascendencia del enfoque interdisciplinario a uno transdisciplinario para alcanzar, así, lo que Jerome Bruner (2000) ha llamado "reinventar la cultura de la educación". Comprendemos, por tanto, la educación como acto comunicativo y como interacción entre personas con distintos niveles de conocimiento, que se "sintonizan", convirtiéndose estos actos e interacciones en nuestras unidades de análisis, que se inscriben en diferentes sistemas que influyen en la claridad y el tipo de dicha interacción: la formación del maestro, su grado de seguimiento de una formación continua en servicio, el sistema de gestión y administración, las condiciones del entorno físico, el clima de trabajo, espacios formales y no formales de educación y características orgánicas y psicológicas del estudiante, entre otras similares (Flórez y Moreno, 2006)4. Esta mirada compleja

4 Utilizamos acá el término unidad de análisis en el sentido que le da Vygotski (1995) en su obra: una unidad que refleja un núcleo de la dinámica de las actividades analizadas. No se trata entonces de nos conduce a la necesidad de pensar y exponer el significado real del conocimiento, las relaciones complejas entre aprendiz-experto, y los procesos autoorganizativos que se juegan entre aquellos que conocen y los ambientes escolares.

El artículo se organiza en torno a cuatro apartados. El primero presenta una breve reseña histórica de los conceptos que han servido como base a la idea de la complejidad. El segundo, expone, de manera concisa, algunas nociones centrales de las teorías de la complejidad. La tercera sección desarrolla la relación comunicación-lenguaje-educación vista desde los postulados de estas teorías. La cuarta y última sección presenta una síntesis de los conceptos que comenzamos a elaborar en este texto.

\section{Nacimiento de las explicaciones desde la complejidad}

Durante siglos, el ser humano ha tratado de comprender la organización de la naturaleza que lo rodea. Los primeros pensadores, como en el caso de Platón, veían, curiosamente, el mundo como una totalidad con un orden y una organización en los que cada una de las partes se hallaba en concordancia con las demás: un mundo en perfecta armonía. Además, para filósofos como Aristóteles, los elementos de este mundo mutaban o se modificaban gracias al movimiento de sus partes. La totalidad que significaba este mundo permanecía perenne, inmodificable, solo la disposición de las partes se alteraba o destruía. Es así que, con estas consideraciones filosóficas iniciales sobre la organización del mundo, se daba comienzo a las primeras concepciones de lo que hoy se concibe como la idea de sistema y, en particular, con la idea de sistema complejo.

Un sistema es, en esencia, un conjunto de cosas, elementos y variables que mantienen ciertas relaciones entre sí de manera ordenada y de cuya interacción resultan propiedades emergentes, no reducibles a los elementos o variables constituyentes. La concepción de sistema tendría implicaciones importantes en la física y en la biología, en particular en la comprensión de los llamados sistemas físicos (como el sistema solar) o en los sistemas biológicos (como el sistema nervioso o digestivo). Sin embargo, sus aplicaciones en el campo de lo psicológico o social resultaban menos evidentes.

la división en elemento producto de la descomposición según un paradigma teórico, porque ha perdido significación al separarlo de otros elementos con los cuales conforma una totalidad. 
Una de las primeras aproximaciones en la comprensión de los sistemas, desde la biología, provino de la mano del biólogo alemán Ludwig von Bertalanffy. Este autor, por medio de su Teoría General de Sistemas (TGS), estipuló los principios sobre los que se soportaría cualquier generalización del concepto de sistema, principios que incluían una clasificación o tipología y una organización de sus propiedades. Bertalanffy (2000) señala que las propiedades de los sistemas no pueden ser descritas, de forma significativa, con base en el análisis de sus elementos separados. Con esto resalta la necesidad de interpretación de los sistemas de forma holística y sintética, interpretación que, como veremos, contraviene los principios, ya tradicionales, de una ciencia puramente analítica. La comprensión de los sistemas sólo sería posible cuando se estudian globalmente, y cuando se involucran todas las interdependencias de los subsistemas que lo conforman.

A partir de la forma de intercambio de energía o materia con el medio, se han establecido en la física varios tipos de sistemas: sistemas aislados, cerrados y abiertos (Rumer y Ryvkin, 1980). En los sistemas cerrados, por ejemplo, se producen intercambios de energía, pero no de materia con el medio ambiente circundante, en cambio, en los sistemas abiertos, se dan tanto intercambios de energía como de materia con el exterior, compensaciones como las que tienen lugar en los organismos vivos. Bertalanffy (1978), en su estudio de los sistemas abiertos, describió dos características fundamentales de este tipo de sistemas, la primera es que estos pueden alcanzar un mismo estado final desde condiciones iniciales diferentes, y por diferentes caminos; a esta propiedad la llamó equifinalidad. La segunda se basa en el hecho de que el mundo de lo vivo exhibe transiciones hacia un orden superior ${ }^{5}$, a esto lo llamó Ley de la Evolución Biológica. En los sistemas abiertos, tendrían un lugar preferente los sistemas vivos, en los cuales, además de intercambios de materia y energía con el medio, también se generarían intercambio de información.

Con la información, se introduciría otro elemento importante que cambiaría el curso de las nacientes teorías de la complejidad y que, para efectos de este documento, tendría un papel importante en el

5 Teleológicamente este incremento del orden biológico se caracteriza por aumento de la heterogeneidad o variabilidad de las especies y un aumento de la complejidad estructural de las mismas. campo educativo. Rescatamos, por tal razón, dos de las clasificaciones de sistemas que se hallan en la jerarquía de complejidad de K. Boulding (1956), en las que se juega, de manera implícita, el concepto de información y que resultan relevantes para el caso: los llamados sistemas humanos y los sistemas socioculturales u organizaciones sociales. Los primeros incluyen sistemas con capacidades de autoconciencia, autosensibilidad y de simbolismo como medio de comunicación, gracias a la capacidad humana de manejo de herramientas simbólicas como el lenguaje. Los sistemas socioculturales, además, representan el conjunto de individuos con la capacidad de crear un sentido social de organización, de compartir cultura, historia y futuro, de disponer de sistemas de valores, de elaborar sistemas de significados. Ambos sistemas, según Boulding, se encuentran en un nivel de complejidad superior al de los sistemas abiertos. Aquí entendemos los sistemas educativos como sistemas socioculturales en los que se comparten conocimientos ${ }^{6} y$ se integran subsistemas de significado y subsistemas humanos.

Adicional a las propiedades y características de los sistemas y a las nociones de información, las nacientes teorías de la complejidad empezaron a incluir otros conceptos que complementaban el sentido general que sustenta las teorías de los sistemas complejos, como: caos, sinergética y homeostasis.

La llamada teoría del caos era la resultante, en un principio, del trabajo de físicos como Henry Poincaré, quien, con su topología algebraica, transformaba la idea de espacio7. Aportes de igual relevancia aparecían, hacia la década del setenta, con el físico Hermann Haken, quien, con su concepto de sinergética, abría paso al estudio de los procesos de formación de las estructuras de los sistemas al disminuir sus grados de libertad: de estructuras caóticas a estructuras estables. Para ello, Haken estableció que un sistema inestable podía hacerse estable mediante la aparición de un parámetro que esclaviza los grados de libertad del sistema, a esto lo llamó principio esclavizador o parámetro de orden (Haken, 1999). La utilidad de las ideas de Haken

6 Aquí entendemos por conocimiento tanto el estado de quien conoce o sabe algo, los contenidos sabidos o conocidos, como el proceso de conocer.

7 Del trabajo de Poincaré deviene, en parte, los desarrollos de la teoría de conjuntos de Julia y, posteriormente, los famosos aportes de Mandelbrot a la comprensión de las estructuras fractales. 
radicaba en posibilitar el estudio de las propiedades de los sistemas complejos que no se hallan en sus etapas iniciales sino en estados posteriores surgidos como consecuencia de la cooperación e interrelación entre sus partes.

Otro concepto de igual importancia, que emerge en el océano de la complejidad, es la idea de homeostasis, del biólogo Walter Canon. La homeostasis es la capacidad de los seres vivos para mantener sus constantes vitales en unos límites que los hacen viables por medio de procesos de retroalimentación. Con la homeostasis, era posible medir la proporción entre el valor de los cambios del entorno y el valor de los cambios en la estructura (interna) del sistema. Cuando un sistema posee un alto índice de homeostasis, éste transforma significativamente su estructura ante cambios del entorno, permitiéndole en muchos casos, la superviviencia.

Estos primeros conceptos abrirían un panorama más amplio en la comprensión de la naturaleza. El nuevo paradigma saltaba de un atomismo centrado en las partes constituyentes a un interaccionismo que daba lugar a sistemas emergentes. Los nuevos niveles de abstracción en la naciente ciencia de la complejidad incluían expresiones referidas a un crecimiento irregular por medio de cursos evolutivos no lineales, entreveramientos y sensibilidades a las condiciones iniciales. Dicho paradigma tuvo impacto entonces en distintos campos de las ciencias sociales, por medio del uso de conceptos como sistemas educativos, emergencia del lenguaje e interacción comunicativa compleja. A continuación se hará referencia a algunos conceptos básicos de este paradigma.

\section{Conceptos básicos de las teorías de la complejidad}

Del pensamiento en complejidad alcanzamos a derivar dos ideas fundamentales: la primera es la necesidad del abordaje de un fenómeno desde todos los componentes implicados en él, es decir, la importancia de comprender todas las posibles relaciones que se plantean en la dinámica de funcionamiento del fenómeno, con implicaciones en diversos niveles, para dar cuenta, de manera integrada, del fenómeno mismo. La segunda idea es la invitación a observar los fenómenos haciendo caso omiso de los límites entre disciplinas y paradigmas. Esto plantea la importancia de no hacer observaciones y explicaciones parciales de los fenómenos, restringiendo la mirada a un único campo disciplinar o teoría. El abordaje de los fenómenos debe tener en cuenta todas las relaciones que estos proponen, haciendo uso del mayor número de posibles herramientas de investigación existentes para dar cuenta de él.

Lo que percibimos es que el pensamiento en complejidad, en vez de generar respuestas, ha planteado nuevas preguntas derivadas de la necesidad de entender la esencia que convierte al todo en algo diferente de la suma de sus partes, es decir, de la necesidad de comprender la aparición de las propiedades emergentes que posee el sistema en su conjunto, y que no son específicas de ninguno de sus elementos o componentes. Mencionamos a continuación algunas de estas propiedades, de la característica esencial de todo sistema complejo de dependencia que va: de un estado con respecto a su estado anterior.

\section{Autoorganización}

Todo sistema se inscribe en el tiempo, y en él es común que se formen nuevas organizaciones y nuevos elementos no presentes ni preexistentes. Lo anterior es el resultado de los efectos de la interacción de los elementos que constituyen el sistema. Esta diferenciación de un sistema respecto al emergente y la nueva organización misma son la consecuencia de tres procesos distintos: primero, del efecto de "masa crítica", es decir, cuando el sistema alcanza cierto nivel de desarrollo energético, material o informático, que asegura su cambio; segundo, por razones adaptativas, cuando es necesario el cambio para afrontar situaciones nuevas frente a un entorno planteado; y tercero, cuando existe la posibilidad de construir formas más complejas que la existente; a todo lo anterior se le ha dado el nombre de epigénesis, como proceso de "sintonización" mediante el cual cada individuo (o sistema) se adapta al entono de manera eficiente a partir de las capacidades que posee.

Es importante mencionar que en los procesos de autoorganización no hay pérdida de información, hecho común en la transmisión informacional (p. e., en ciertos actos comunicativos). La autoorganización se sustenta en el concepto de iteración, es decir, de la repetición de ciertos pasos o procesos, 
lo que garantiza que esta perdida de información no suceda. En consecuencia no existe, necesariamente, en los sistemas autoorganizados, un final preestablecido.

La nueva trayectoria que sigue el sistema en el tiempo puede ser tan estable como la anterior o bien llevarle a un colapso. En este último caso, hablamos de bifurcaciones catastróficas. En éstas no cambia la estructura del sistema, sino que, llegado a un punto crítico del mismo, modifica su trayectoria hacia un nuevo atractor ${ }^{8}$ o punto de confluencia. Un aspecto significativo de este comportamiento es la ausencia de señales de aviso o de alarma que nos informen de la proximidad de una bifurcación en base a la historia del sistema. Tampoco los cambios en el entorno nos pueden anticipar la llegada a una bifurcación, ya que las mismas circunstancias del entorno observadas en el momento de la bifurcación pueden haberse dado en etapas anteriores del sistema sin repercusiones.

\section{No linealidad}

Por lo anterior, podemos decir que todo sistema complejo se caracteriza por procesos autoorganizantes y por la emergencia de nuevas estructuras a partir de la interacción de los elementos constituyentes. Cuando hablamos de no linealidad, hacemos referencia a que los efectos de las variables o de las fuerzas en un sistema no pueden describirse como la suma de estos efectos (Van Geert y Steenbeek, 2005a), así todo sistema complejo, en principio, poseerían relaciones multiplicativas (no lineales) en lugar de aditivas (lineales). Cuando observamos un sistema en el transcurso del tiempo, podemos ver que grandes cambios son el resultado de pequeñas diferencias iniciales (Thelen, 2005), lo que establece que en las realidades a estudiar no existe un patrón de crecimiento uniforme en todos los momentos; por el contrario, se contempla la posibilidad que se presenten procesos irregulares propios de su

8 Entiéndase "atractor" como el punto o conjunto de puntos que mueven hacia sí todos los demás elementos y comportamientos del mismo sistema. Este tiene íntima relación con el concepto de iteración, juntos conducen a la idea de fractal, la cual se sustenta en el principio de la autosimilitud como complejidad infinita en un espacio finito. Pensemos, como ejemplo, en un huracán: la fuerza del huracán converge en un punto llamado ojo del huracán o vórtice. Este vórtice funcionaría como punto atractor y estaría determinado por la fuerza misma de la corriente eólica. organización en un momento determinado. La no linealidad también implica que causas simples pueden conllevar a resultados complejos.

\section{Entreveramiento}

Aquí entendemos el entreveramiento como la organización por conformación de relaciones e interacciones más complejas cada vez, lo cual quiere decir una diferenciación de elementos, subsistemas y relaciones entre elementos de muy diverso tipo, efecto de la autoorganización. Por medio de éste, se pueden generar estructuras a multiescala o estructuras que siguen un patrón de inclusión sucesiva de sistemas en otros, por medio de distintos niveles jerárquicos de organización.

El entreveramiento es útil en la observación simultánea de los distintos niveles en los cuales se estudia un fenómeno, así, por ejemplo, la comprensión de fenómenos complejos, como los fenómenos mentales, abarcaría el estudio meticuloso de los procesos neurales y subneurales hasta la comprensión de los sistemas sociales y culturales que intervienen en dicho fenómeno mental. El análisis del paso del plano interpsíquico al intrapsíquico en la interacción social que conforma los procesos psicológicos superiores en Vygotski (1979), la modificación de la estructura de las conexiones neurales en el cerebro como producto de la actividad entendida como aspecto funcional del sistema nervioso en Luria (1978) y el crecimiento del niño en función de su inserción en distintos nichos ecológicos de crecimiento en el planteamiento de Bronfenbrenner (1979) son solo algunos ejemplos de la utilidad de este concepto para la comprensión de la interacción en diversos niveles que se dan en los fenómenos educativos. Sólo cuando se tienen en cuenta estos diversos niveles o escalas explicativas es posible tener una mirada más amplia del objeto de estudio.

Tenemos, entonces, que las teorías de la complejidad caracterizan a los fenómenos de acuerdo con una concepción holística de éstos, de su descripción y explicación en términos de totalidad autoconstituyente o autoorganizada, con propiedades emergentes y estructuras entreveradas y a multiescala que surgen como consecuencia de la autoorganización. Esta mirada tendría consecuencias profundas en la epistemología y en la reevaluación de los métodos tradicionales de las ciencias. 
En la historia de la ciencia, con los planteamientos de Descartes, se comenzó a privilegiar un enfoque analítico caracterizado por la tendencia a dividir los fenómenos complejos en partes. Esto significaba reducir la realidad estudiada a un conjunto de elementos y procesos tan simples como fuese posible. Producto de este enfoque es el método analítico, cuyo principio fundamental es "el todo es igual a la suma de sus partes". Fue precisamente este método el que mantuvo lejana la idea del estudio de las complejidades. Sin embargo, su uso permitió un avance importante en la investigación científica hacia finales del siglo XIX.

A finales del siglo XIX y principios del XX, aparece el pensamiento estructuralista ${ }^{9}$ en el que "el todo llega a ser más que la suma de sus partes". Esta corriente trata de dar respuesta a los interrogantes que surgen del estudio de fenómenos que no eran fácilmente analizables o explicables desde el método reduccionista. Las ciencias biológicas y las ciencias sociales, a principios del siglo pasado, vieron la necesidad de otro enfoque: uno que privilegiara el estudio de las relaciones que existen entre los elementos y la diversidad de procesos que se generan. La necesidad de interpretar los individuos y sus relaciones como un todo que va más allá de la suma de los individuos: tal era el caso de los individuos y sus relaciones en las instituciones sociales que emergían como una estructura social, y del organismo como un todo, en el cual, del resultado de las interacciones en este, aparece la vida tal y como la conocemos.

Estas nuevas "visiones" condujeron a una nueva forma más holística de comprensión de la realidad, que tendría repercusiones en el campo de la educación, del lenguaje y la comunicación; algunas de estas implicaciones las encontramos en los trabajos iniciales de Paul van Geert (1991), Alan Fogel y Esther Thelen (1987), y más recientemente en el campo de la educación con las Conferencias sobre Ciencia de la Complejidad e Investigación

9 El pensamiento estructuralista aquí mencionado se diferencia del estructuralismo como movimiento que surge a mediados de la década del cincuenta, a partir del trabajo de Lévi-Strauss, y posteriormente con los desarrollos de Foucault y Lacan entre otros. Este pensamiento estructuralista se caracteriza por figuras como Saussure con su estructuralismo lingüístico, Köhler y su estructuralismo gestáltico, Piaget y su estructuralismo psicológico y el emergentismo social de Durkheim.
Educativa, realizadas en 2004 en la Universidad de Alberta en Canadá. Producto de estos avances es la integración que presentamos a continuación entre las teorías de la complejidad, la comunicación y la educación, objeto del presente escrito.

\section{La relación entre comunicación y educación en el marco de la teoría de la complejidad}

Partimos del postulado según el cual el objeto nuclear de estudio de la educación es el aprendizaje (Hederich, 2007). Toda cultura ha creado formas para que sus miembros aprendan, ya que esto es fundamental para que su tradición sea sostenida y prolongada en el tiempo. Desde este punto de vista, la educación tiene como condición las situaciones de instrucción conformada por el conjunto indivisible de tres elementos: la forma particular de las tareas, las características específicas de quien enseña (estables y del momento) y las características de quien aprende (estables y del momento) ${ }^{10}$ (Saracho, 2003).

10 Utilizamos como punto de partida la definición que hace el Diccionario de la Real Academia de la Lengua Española sobre los siguientes términos, en este punto y a lo largo del texto:

Educación: acción y efecto de educar. Crianza, enseñanza y doctrina que se da a niños y jóvenes. Instrucción por medio de la acción docente.

Educar: dirigir, encaminar, adoctrinar. Desarrollar o perfeccionar las facultades intelectuales y morales del niño o del joven por medio de preceptos, ejercicios, ejemplos, etc. Educar la inteligencia, la voluntad. Desarrollar las fuerzas físicas por medio del ejercicio, haciéndolas más aptas para su fin. Perfeccionar, afinar los sentidos. Educar el gusto.

Instruir: enseñar, adoctrinar. Comunicar sistemáticamente ideas, conocimientos o doctrinas.

Comunicación: acción y efecto de comunicar o comunicarse. Trato, correspondencia entre dos o más personas. Transmisión de señales mediante un código común al emisor y al receptor.

Lenguaje: conjunto de sonidos articulados con que el hombre manifiesta lo que piensa o siente. Lengua (sistema de comunicación verbal). Manera de expresarse. Lenguaje culto, grosero, sencillo, técnico, forense, vulgar. Estilo y modo de hablar y escribir de cada persona en particular. Uso del habla o facultad de hablar.

Aprendizaje: acción y efecto de aprender algún arte, oficio u otra cosa. Tiempo que en ello se emplea. Psicol. Adquisición por la práctica de una conducta duradera.

Aprender: adquirir el conocimiento de algo por medio del estudio o de la experiencia. Tomar algo en la memoria.

Nótese que en este nivel de las definiciones no técnicas, también se liga a la educación (por medio de la instrucción) con la comunicación, como parte de un mismo conjunto de fenómenos. 
Los tres elementos de la unidad, anteriormente descrita, nos permiten analizar el grado de distancia o de proximidad entre aprendices, entre quien enseña y los aprendices, y entre los aprendices y el material a aprender. No debemos olvidar otro factor fundamental de todo acto educativo que enfoca el aprendizaje: es una forma de transmisión cultural. En su desarrollo, esta forma plantea diferentes momentos de la relación entre participantes, de acuerdo con B. Bernstein (1989): 1. Introducción al círculo de aprendices y enseñantes, 2. Afirmación del conocimiento y de las habilidades adquiridas en el grupo y 3. Evaluación y paso por rituales de iniciación para pertenecer a una comunidad con un estatuto mayor a los que inician o a los que están en niveles anteriores.

La introducción al círculo de aprendices y enseñantes hace referencia a las formas que se usan para que una persona se acerque por primera vez a la práctica de un conocimiento, con el cual comienza a formar parte de la comunidad de aprendices. Puede pensarse, por ejemplo, en los niños que inician la escuela primaria, en los trabajadores de la fábrica que se inician en un oficio nuevo o en los estudiantes que acaban de ingresar a la universidad. En dicha introducción, se hace que "los nuevos" se aproximen a las habilidades a aprender. También se ejercitan en la habilidad con la guía del maestro o el experto (Rogoff, 1995).

La afirmación del conocimiento y de las habilidades adquiridas en el grupo hace referencia al mutuo enriquecimiento que fortalece los conocimientos y las habilidades por hacerlos patrimonio del grupo. En la perspectiva de cada grupo, estos conocimientos, habilidades y actividades lo delimitan con respecto a otros grupos particulares de personas con otras funciones en la comunidad (Jodelet, 1991). Son los conocimientos que hacen, por ejemplo, identificar a los estudiantes como tales y adquirir una serie de conocimientos de acuerdo con la relevancia que estos tiene sobre la vida del grupo y sobre la dinámica particular de cada uno de ellos en los distintos círculos de su vida.

Por último, la evaluación hace referencia a los puntos en los cuales los aprendices realizan los rituales propios de la iniciación y transición en ciertas comunidades más específicas en su aprendizaje, llamados también ritos de iniciación: a la comunidad de médicos, a la comunidad de especialistas en educación o a cualquier otra.
Se sigue entonces que la educación es parte de la cultura, esto es, de la conformación y evolución de toda tradición. La educación se entiende como una forma en la cual las personas se introducen en la cultura mientras participan activamente en ella, o mejor, mientras los elementos de la tradición forman parte de su mundo de intercambios significativos ${ }^{11}$ (Bruner, 2000). En este sentido, es preciso que entendamos que la educación está presente en las instituciones escolares y académicas, pero también está en otros ámbitos fuera de dichas instituciones. La educación, en la definición que de ella da el Diccionario'12, lleva a ver la manera en que los aprendices entran en el mundo propio de los conocimientos impartidos y no sólo en cómo se les "entusiasma a verlos". Se trata entonces de una situación con una guía interpersonal. Por ello, en toda situación educativa hay una puesta en juego de las capacidades que tiene cada persona (Bruner, 2000). La pregunta para los investigadores en educación y para los educadores en ejercicio sería: ¿cómo hacer funcionar dichas capacidades en los actos educativos?

La respuesta parece provenir de un paradigma que enmarque las capacidades de los aprendices bajo un modelo sistémico, que visualice de manera activa la interacción entre el aprendiz y el maestro, y que conduzca a una comprensión compleja de los ambientes o espacios de conocimiento. El paradigma de la complejidad es el marco de referencia para entender estos tres componentes bajo una mirada comunicativa por medio de tres elementos esenciales que se presentan a continuación: las estructuras de conocimiento, la interacción educativa-comunicativa, y los espacios de conocimientos.

\section{Estructuras de conocimiento}

Las estructuras de conocimiento, desde el marco de las teorías de la complejidad, tienen relación con lo que se ha llamado desde hace algún tiempo pensamiento complejo, el cual deriva de las ideas del

11 Ya hacía tiempo, escritores como George Orwell (1961) realizaban planteamientos en este sentido, cuando mostraba la apropiación de recursos de la cultura por medio de la literatura y de las historias populares.

12 Ver definiciones de educación y educar en la anterior nota a pie de página. 
filósofo y sociólogo francés Edgar Morin (1996), y que es posible resumir como la generación de cierto tipo de prácticas pedagógicas capaces de desarrollar en el aprendiz una visión del mundo más compleja y holística, permitiéndole pasar de la incertidumbre del mundo a la certidumbre por medio de la adquisición de información o conocimiento. No es el propósito del presente escrito ahondar en las características del llamado pensamiento complejo. Por el contrario, esperamos tomar otra ruta un poco distinta de la postura moriniana para pensar el desarrollo de estructuras de conocimiento complejas como procesos autoorganizativos que devienen de la dinámica interactiva de los aprendices con los maestros.

Sabemos que la emergencia de nuevos desarrollos sobre el conocimiento de las personas depende de fases de autoorganización de los mismos. Como mencionamos en párrafos anteriores, la autoorganización refiere a procesos que tienen lugar en el tiempo y que se caracterizan por la aparición de nuevas estructuras resultantes de la sensibilidad a las condiciones de partida de las relaciones. Un ejemplo de estos procesos autoorganizativos lo observamos en un trabajo anterior de investigación, en el cual algunos niños en el preescolar no daban aún muestras claras de distinguir entre números, letras y dibujos; con una breve exposición a algunas láminas, los niños adquirieron este conocimiento casi como si se "hubiesen disparado" (Flórez, Torrado, Rodríguez y Quintero, 2003). La emergencia de estas capacidades ya la hemos referido en otros textos (Castro y Flórez, 2007) y está vinculada, en primera medida, a efectos de masa crítica o acumulación de información o experiencia, o en segunda instancia a pequeñas modificaciones producto información novedosa, que conduce a cambios significativos de los sistemas de conocimiento. El caso mencionado nos muestra que los niños tenían habilidades y conocimiento previos suficientes para conocer las notaciones: apenas fue suficiente un pequeño trabajo conjunto para desarrollar este conocimiento, que se consolidó casi como en una serie de "efectos en cadena".

Asimismo, en los procesos de autoorganización existe la necesidad de organizar y volver a desorganizar las estructuras de conocimiento con el fin de tener capacidades de cambio para afrontar nuevas situaciones planteadas en el entorno. Ejemplo de ello es el modelo de explicación del desarrollo en la teoría-teoría (To-To), formulada por Gopnik (1999). Allí se propone que el desarrollo cognitivo consiste en el cambio de los marcos de explicación de los fenómenos, lo cual se logra con ayuda de recursos avanzados y elementales, como las generalizaciones empíricas, el ensayo y error, la reformulación y la ayuda del lenguaje y el nuevo vocabulario. Como hemos mencionado, la autoorganización implica también procesos nacidos del momento inmediatamente anterior de organización. El conocimiento en un momento dado puede avanzar de acuerdo con lo detalles que presenta justo antes de su transformación en el desarrollo y el aprendizaje ${ }^{13}$ (Gopnik, 1999; Karmiloff-Smith, 1992).

De igual manera, la autoorganización, necesita de procesos iterativos para garantizar que no haya pérdida de información importante (ver, por ejemplo, Karmiloff-Smith, 1992). De ello surge la necesidad de volver sobre el mismo material con nuevas preguntas e interrogantes nacidos de la misma actividad de los aprendices. Por ello, es importante plantear, en las actividades dirigidas al aprendizaje, situaciones de resolución de problemas que permitan desplegar las capacidades por medio del razonamiento en el curso de la actividad propuesta (Puche-Navarro, 2003). Esto se basa en la idea según la cual el proceso educativo es una "dialéctica" entre las elaboraciones que el sujeto hace sobre su propio conocimiento en interacción con la acción intencional educativa que hace el maestro como creador de actividades dirigidas al aprendizaje de los estudiantes.

De lo anterior, se sigue la necesidad de usar todas las vías que privilegien distintas maneras de asimilar la información, para poder llegar al mayor número de alumnos y esquemas de conocimiento posibles en la audiencia de estudiantes (Hederich y Camargo, 2002). Por ejemplo, se puede presentar la misma información en forma de esquemas, relatos, explicaciones, o de forma gráfica. Esto, a su vez,

13 Esta idea está inspirada en el modelo matemático de las sucesiones de Markov. De acuerdo con este modelo, el estado actual de evolución de un sistema depende primordialmente de el estado de evolución inmediatamente anterior en el mismo sistema (Puche - Navarro, 2005). 
subraya la importancia de una evaluación que guíe la formación y que no sirva solo como elemento de sanción.

Igualmente, en cada situación de aprendizaje en un contexto educativo es preciso contar con el factor tiempo. Éste viene dado, además de la duración de los procesos, en la intensidad del uso del mismo, en el ritmo del aprendizaje y en la secuencia que se da a éstos de acuerdo con una lógica que exigen los contenidos y procesos de asimilación (Hederich, Camargo y Reyes, 2005). Esto es de especial importancia en el trabajo con poblaciones llamadas "vulnerables" o en inclusión; un factor importante para su aprendizaje exitoso es el conocimiento de su ritmo, manera y condiciones particulares (Guido, 2006).

Las condiciones iniciales marcan los momentos en los cuales se muestra la importancia e interés que pueden tener los distintos tipos de conocimiento. En tales momentos, se comienza a crear la forma de una relación con el conocimiento y con la persona que lo imparte, la cual se hace fundamental para los aprendices. Aunque no sea un factor que determine todo el curso siguiente de evolución en cada aprendizaje, su importancia radica en la señalización que comienza a hacer de aquello que es importante en un área de conocimiento, mostrando la relevancia 14 , como es posible de desplegar en cualquier acto comunicativo entre seres humanos.

Como corolario de la organización de la estructura de conocimiento en el aprendizaje y el desarrollo, dicha estructura tiene un incremento de orden e información. Por ello, el conocimiento construido en ésta es más complejo que la información que se presenta, la cual interactúa siempre con un conocimiento ya organizado y jerarquizado por la misma estructura. Ello hará que sea más o menos importante la información para lograr cambios significativos que posiblemente no se hayan previsto.

14 Acá hacemos uso del término relevancia tal y como se usa en la teoría de la relevancia (Sperber y Wilson, 1982): una serie de aspectos que el emisor resalta intencionalmente en su mensaje para que su interlocutor centre su atención en ellos, y poder acordar así lo que de un símbolo cualquiera se transmite a un interlocutor. Esto permite prever sus reacciones para actuar en consecuencia con ellas y poder mantener el contacto y el flujo de la comunicación.

\section{Interacción educativa-comunicativa}

Como hemos visto, las teorías de la complejidad asumen la importancia de ver las totalidades más que la suma de los elementos que la componen, poniendo un especial énfasis a las interacciones que se generan entre estos elementos. Si bien, ésta es una de las diferencias radicales con las vertientes reduccionistas tradicionales, para un teórico de la complejidad son las interacciones en un ambiente, un campo o, en general, de un sistema dado, las responsables por la aparición de los fenómenos, las propiedades y conductas que caracterizan al sistema estudiado.

En los sistemas de educación, encontramos diversas formas de relación de cada uno de sus miembros. Diferencias en la relación entre pares o en la relación maestro-alumno. Esta interacción tiene un papel importante en la construcción social y de los individuos. En ella se juega la transmisión de conocimiento y la transmisión cultural. En el contexto educativo todo acto necesita de este intercambio entre aprendices y enseñantes. Dicho intercambio es posible sólo por medio de una sintonización entre unos y otros, donde uno proponga, el otro reciba y a su vez de una respuesta con base en la cual vuelva a reaccionar el primero y se cree entonces un nexo que llamamos comunicación (Clark, 1998). Las maneras en las cuales se plantea este intercambio son por medio del lenguaje, específicamente por medio de la conversación, que tiene en cuenta la suposición de conocimientos al interlocutor en un momento dado. En la conversación se ponen en común los conocimientos, y esto exige organizarlos para hacerlos claros al interlocutor. Es una actividad conjunta que no es reducible a uno sólo de sus participantes 15 .

En la educación se juegan los procesos de instrucción por medio de actos comunicativos (actos del lenguaje), entender la evolución en el tiempo

15 Cabe aclarar que se da una comunicación cuando se llega a un acuerdo de lo que se quiere decir entre quienes participan en un intercambio de ideas (Clark, 1998). En este sentido, hay sintonización cuando se ha llegado a este acuerdo. Todo ello implica que quien enseña tenga claridad sobre la manera en la cual el aprendiz está comprendiendo las ideas o habilidades propuestas, para cooperar con él de manera más precisa. A su vez, esto exige contar con métodos de indagación que sean precisos y adecuados a cada situación para cada grupo de aprendices en los que se pueda presentar. 
tanto de las estructuras como de los procesos permite la comprensión del porqué de la aparición de momentos de ruptura frente al conocimiento que se imparte. En esta relación hay tres elementos clave: el sentido de la interacción, la influencia reciproca de la interacción y la influencia reciproca en el sistema de relaciones.

Por lo anterior, se asume que la educación está mediada por actos comunicativos. Cobran un lugar de fundamental importancia la transmisión oral, $\mathrm{y}$ otros recursos como las actividades de lectura y escritura, que en sí mismas también son objeto de la enseñanza: de ellas depende todo éxito en otros aprendizajes por ser éste el canal de comunicación (Flórez, Arias y Guzmán, 2006). En esta relación, es importante tener en cuenta que las mismas capacidades de los aprendices -sobre todo de los niños- están en relación con el estado y con el cambio cualititativo en sus capacidades, lo que se conoce como desarrollo. Todo aprendizaje (entendido como modificación permanente en la manera de realizar las actividades) depende de la pauta que dé el desarrollo; de la misma manera, todo desarrollo se ve nutrido por distintos aprendizajes, ya sean incidentales o intencionalmente promovidos por el adulto. Esta relación es posible gracias a que el acto de conversación permite la adecuada interacción entre enseñantes y aprendices. En la conversación, el lenguaje obliga a sus usuarios a organizar los conocimientos de forma clara, precisa y secuenciada para presentar las ideas a los demás (Vygotski, 1979, 1995; Bruner, 2000). De esta manera, la educación es comunicación -intercambio permanente de mensajes- e interacción -acto en el cual se informa a una persona en la acción conjunta, mientras la otra persona se forma y el emisor se forma también a sí mismo- (Jörg, 2005).

De forma similar, de acuerdo con el modelo que venimos presentando, el conocimiento cambia y se complejiza con el tiempo en una medida que puede no ser siempre la misma en todo momento. La relación tripartita entre enseñantes, aprendices y tareas no tiene en todos los casos un mismo producto como efecto de sus interacciones: es distinto según las características que adquieren en cada momento de la interacción (Jörg, 2005, Vygotski, 1979, Saracho, 2003). El resultado no es totalmente previsible, ni está completamente determinado de antemano, lo que significa que el aprendizaje de cada persona depende de una probabilidad dada la interacción de cada una de estas variables.

Como efecto de la organización, en la mutua alimentación entre aprendizaje y desarrollo se generan elementos nuevos no presentes ni preexistentes en los conocimientos. Estos elementos emergentes no están contenidos desde antes en el conocimiento previo ni pueden explicarse, reduciendo el fenómeno a constituyentes aislados de él. Así, en el acto educativo, éste no puede reducirse sólo al aprendiz, sólo al enseñante, o sólo al tipo de tareas que deben afrontarse en el aprendizaje: que un niño comprenda las operaciones básicas de la aritmética no depende sólo de las características del profesor (como se suele pensar entre la mayoría de personas) ni solamente de las características de los estudiantes (como con frecuencia se piensa entre los maestros) ni solamente del tipo de tareas y de la bondad de los métodos que se aplican en sus enseñanza (como se piensa con frecuencia entre los expertos estudiosos de la didáctica de áreas específicas). Depende de todas ellas, con un mayor o menor énfasis en algún aspecto, dado el carácter de la interacción entre los elementos implicados en el aprendizaje en un momento particular. El lenguaje, vía principal de esta interacción, logra una forma de objetivación de la realidad que permite a los actores de la interacción descentrarse de su perspectiva particular y puramente subjetiva y, a la vez, les obliga a organizar su propia perspectiva para expresarla de manera lingüística a los demás (Cabrejo, 2004).

\section{Espacios de conocimiento}

Sin embargo, el juego de las estructuras de conocimiento y de las interacciones comunicativas-educativas sólo es posible de entender en el marco de los espacios de conocimiento, es decir, en la comprensión de los sistemas informativos-culturales, en el que las entidades de aprendizaje intervienen con sus necesidades y prácticas. Con el concepto de sistema, vemos una primera aproximación a estos ambientes educativos como sistemas socioculturales abstractos ${ }^{16}$, sistemas de intercambio de información en

16 El mismo Bertalanffy hace una diferenciación entre sistemas reales y abstractos. Por sistemas reales se entiende las entidades percibidas o deducidas de la observación, cuya existencia es independiente del observador. Un sistema vivo se ajusta a esta característica. Por sistemas abstractos se concibe un sistema conceptual que tiene correspondencia con la realidad, pero cuya existencia depende de su relación con el observador (von Bertalanffy, 2000). 
los que se imponen ciertos procesos y estructuras, posibles de comprender bajo una mirada a multinivel, claramente facilitada por la perspectiva de la complejidad.

Figura 1. Espacio de conocimiento como lugar de intercambio entre aprendiz-experto.

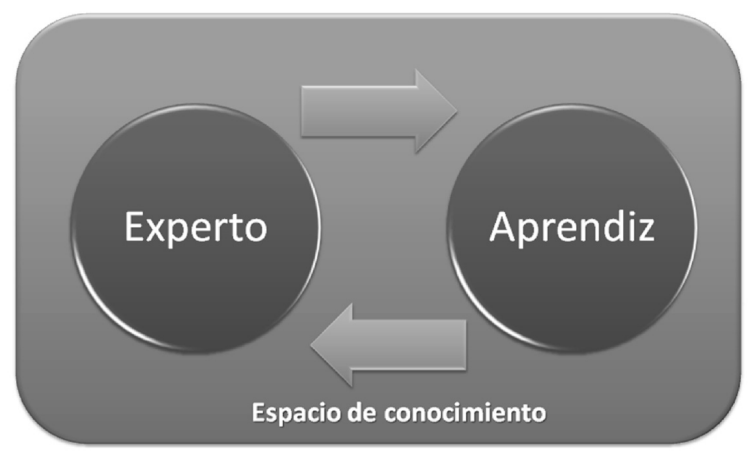

Lo que aquí llamamos procesos está mayoritariamente relacionado con los niveles macro del sistema informativo-cultural, especialmente vinculados al nivel social. En este punto, se entiende que no se concibe una división estricta y tajante entre aspectos individuales y sociales porque en cada individuo se entrecruzan diferentes relaciones sociales producto de la interacción. Los cambios a este nivel son dependientes de las modificaciones en las relaciones o interacciones entre las estructuras de conocimiento. Se habla entonces de "procesos educativos" y se interpretan durante el desarrollo de una historia -o de un tiempo específico-. Las estructuras, por su parte, son más dependientes de los niveles micro o de los niveles individuales (al cual pertenecen las estructuras de conocimiento). Los cambios a este nivel se dan, por ejemplo, en (más, no entre) el aprendiz o experto. En ellos la estructura de conocimiento llega a ser altamente variable.

El sistema educativo, como espacio de conocimiento o macroestructura, sufre de procesos de complejización similares a los que se llevan a cabo en las estructuras de conocimiento. Es decir, a medida que las redes de comunicación en la macroestructura se extienden, nuevos elementos ingresan al sistema; así, el sistema se complejiza en la medida del número de microestructuras intervinientes. De forma paralela, cada parte de este sistema encuentra, a su vez, un proceso de complejización individual. La complejización, por tanto, abarcaría las modificaciones en un grupo social, visto anteriormente, en la modificación organizada de las redes de aprendizaje del grupo o de las redes de interacción y, por otro lado, en el nivel de las partes que componen al macrosistema como modificación organizada de los individuos y manifiesta en los cambios de las estructuras de conocimiento de cada uno de ellos, por una parte, y por otra en alteraciones de las estructuras cognitivas, entendidas como modificaciones en los esquemas cognitivos o en el contenido del conocimiento mismo que se juega en las interacciones comunicativas.

De este modo, la interrelación (interpsíquica) llega a establecerse entre esferas macro de las instituciones y esferas micro de los niveles intrapersonales. Esta interrelación establece un juego entre lo individual y lo social, único de percibir bajo una mirada compleja. En este devenir inter-intra encontramos como referencia la conocida figura de la banda de Möbius. En ella se ilustra el movimiento desde lo individual (un lado de la banda) y su continuidad hasta ser parte de lo social (el otro lado), ejemplificándose el paso del individuo a la sociedad y viceversa, ya que lo social también se transforma en individual. No existe, entonces, en este proceso, una división estricta entre lo individual y lo social, sino que son campos interrelacionados y no divisibles en la constitución de cada individuo y de sus relaciones con otras personas.

Figura 2. La banda de Möbius muestra el paso de lo individual a lo social y viceversa.

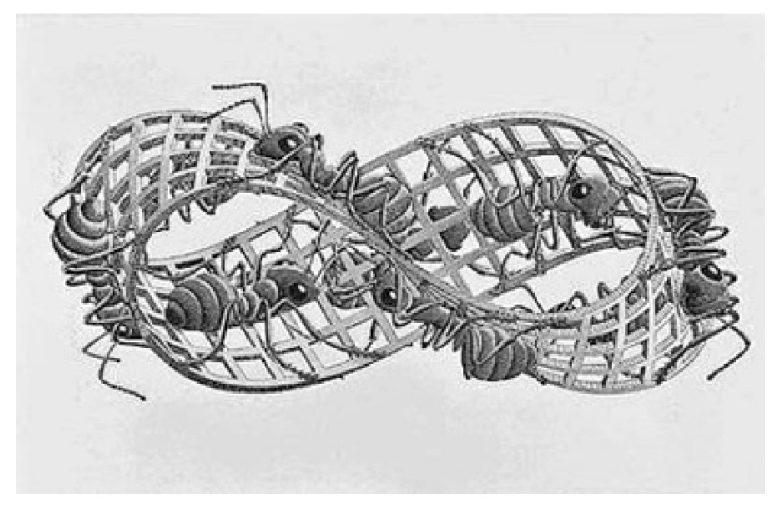

Lo que aquí exponemos es una noción de la realidad, no como una representación que da cuenta total de ella, sino como una matriz generativa que permite comprender varios casos posibles que en ella se enmarcan. En el caso de la interacción y la comunicación en educación, esto tiene como 
consecuencia desvirtuar la estricta división entre lo social y lo individual en el aprendizaje. Toda persona cuando aprende algo lo hace con otras personas y asimila internamente los conocimientos. Cuando interactúa con otras personas, razona individualmente y a la vez con los demás. Cuando asimila internamente los conocimientos, lo hace fuera de la actividad con otros, pero evocando la actuación que con otros le ha permitido asimilar los conocimientos que provienen de una tradición de ciertos grupos sociales. Por ello, analizar este proceso de manera "estrictamente individual" o "estrictamente social" nos hace perder una parte importante de la explicación del fenómeno del aprendizaje y de la mediación de éste por un enseñante (Bruner, 2000; Puche-Navarro, 2003).

$\mathrm{Al}$ igual que en los microsistemas o estructuras de conocimiento, los macrosistemas o espacios de conocimiento sufren de procesos de autoorganización que conducen a la emergencia de nuevas relaciones o interacciones y nuevas formas de aprendizaje. La manera en que se dé este ejercicio de autoorganización puede conducir a procesos específicos de retroalimentación de las estructuras de conocimiento, lo cual quiere decir que el conocimiento que ha sido fuente de cambio en la microestructura, a su vez, se modifica y sirve de fuente recurrente de nuevos conocimientos. El juego comunicativo sería el encargado de la transducción informacional que conduce a la aparición o emergencia de nuevos conocimientos, ya sea en la estructura o en el ambiente de conocimiento. Este elemento emergente, a su vez, alimenta la actividad del experto $y$, por un proceso claramente iterativo y transformacional, genera nuevas dinámicas de aprendizaje y conocimiento, más complejas y más ricas.

En estos procesos de complejización también hay momentos de estabilización en los que el sistema de conocimiento (entiéndase el macrosistema, ambiente educativo o ecosistema comunicativo) mantiene sus propiedades. La estabilidad temporal es la que, en principio, facilita el proceso de adquisición de conocimientos porque éstos se muestran asequibles a las estructuras de conocimiento (como conocimiento consolidado y cierto). Para los teóricos de la complejidad, un sistema pierde el momento de estabilidad cuando entra en lo que es considerado una "fase de transición" (como la que se encuentra en la fase del agua de líquido a gas). Éstas son igualmente importantes porque marcan los momentos de reflexión o interrogación de las estructuras. El conocimiento deja de ser completamente cierto y evidente para ser cuestionado, lo cual impulsa la dinámica iterativa a renovarlo o reconstruirlo para luego ser interpelado a una estructura de conocimiento experta, generándose un momento de revolución o de ruptura del microsistema (ver, por ejemplo, Castro, 2007).

El estudio de estas fases transicionales, cómo y cuándo aparecen y qué propiedades específicas emergen permite una mayor comprensión de un fenómeno o realidad específica, como la realidad de la educación, al posibilitar la comprensión de aquello que empuja a la aparición de nuevas formas de aprendizaje, de novedosas formas de reflexión y adquisición de conocimientos, y deja ver qué conocimientos nuevos aparecen al diferenciarlos por medio de la claridad de la transición entre conocimientos previos.

\section{Conclusiones}

Gracias a los distintos avances de la ciencia de la complejidad, ha sido posible entender mucho mejor el panorama de la relación comunicación-lenguajeeducación. Se han abierto las puertas para aclarar el papel de la comunicación y del lenguaje en los procesos educativos. Ahora es posible dilucidar cómo varían y se estabilizan los procesos dependientes del tiempo como son, entre otros, los procesos relacionales, pedagógicos, emocionales o de aprendizaje. Se ha permitido que salgan a la luz las variadas relaciones no lineales en las interacciones entre pares y maestros en los ambientes educativos, y se ha abierto el camino para hablar de conocimiento emergente.

La teoría de la complejidad nos ha permitido, en resumidas cuentas, abordar el fenómeno educativo tomando como unidad los sucesos y las variables implicadas en el acto comunicativo, en el cual participan enseñantes y aprendices que interactúan frente a determinadas tareas. La relación que media entre estos elementos es una relación comunicativa de mutuo intercambio recíproco y que tiene en cuenta la posición o información con la cual el otro parte a cada momento. Por ello, se tiene en cuenta 
la organización de conocimientos por parte de los individuos y entre éstos y sus enseñantes, en la cual media una relación en la que el lenguaje sirve como vía de comunicación e instrumento de asimilación y conducción del saber. Los sistemas (macro y micro), y la interacción entre ellos, llegan a ser sensibles a cada momento del intercambio comunicativo, pero también de las condiciones iniciales que pueden marcar un camino nuevo o la repetición de algo con personas que ya están iniciadas en un cierto aprendizaje. Por lo anterior, resulta relevante atender a los primeros momentos de los intercambios comunicativo-educativo.

Los diversos avances hechos en el marco de relación entre la complejidad-educación-comunicación igualmente muestran la necesidad de una perspectiva evolutiva integradora de las dinámicas causales de la interacción comunicativa para explicar la complejidad de la realidad de la educación. Creemos, al igual que otros autores, que el nuevo

\section{Bibliografía}

Bernstein, B. (1989). Poder, educación y conciencia. Sociología de la transmisión cultural. Barcelona: El Roure.

Boulding, K. (1956). General systems theory: the skeleton of science, en Management Science, 2 (3), 197-208.

Bronfenbrenner, U. (1979). The ecology of human development. Cambridge, Mass: Harvard University Press.

Bruner, J. (2000). La educación puerta de la cultura. Madrid: Aprendizaje Visor.

Cabrejo, E. (2004). Entrar en la lengua, entrar en la cultura. En Fundalectura (ed.), Sexto Congreso Nacional de Lectura. Lectura para construir nación. Bogotá: Editor.

Castro, J. y Flórez, R. (2007). La emergencia del lenguaje y los sistemas dinámicos. Revista Colombiana de Psicología, 16, 185-202.

Castro, J. (2007). Sistemas dinámicos en psicología del desarrollo. Tesis sin publicar. Bogotá: Universidad Nacional de Colombia.

Clark, R. (1998). De la acción al gesto. En A. Perinat (comp.), La comunicación preverbal (pp. 103-142). Barcelona: Avesta. lenguaje de esta perspectiva se debe centrar en una nueva síntesis de las ciencias naturales y las ciencias sociales (Vygotsky, 1978; Jörg, 2005, Mason, 2008). Hacer esto implica que debemos iniciar un proceso de resignificación: repensar la interacción y repensar la complejidad, para ser capaces de reinventar la realidad como dinámica y fluida (Jörg, 2004). Para esta nueva ciencia de la educación, resulta imprescindible repensar la noción de individuo como ente aislado y aislable, y comenzar con la comprensión social de los mismos, de sus interacciones y de sus intercambios interpsicológicos (a la manera de Vygotski, 1979), ubicarlo más allá del paradigma analítico hasta ahora dominante, para mostrarlo como un sistema capaz de generar conocimientos revolucionarios (igualmente en el mismo sentido de Vygotski, 1979) o transicionales, que marcarían las dinámicas futuras de los actos comunicativoseducativos. \}

Flórez, R., Arias, N., y Guzmán, R. (2006). El aprendizaje en la escuela: el lugar de la lectura y la escritura. Educación y Educadores, 9(1), 117-133.

Flórez, R., Torrado, M., Rodríguez, O. y Quintero, C. (2003). Promoción del alfabetismo emergentey prevención de las dificultades de lectura en el aula de preescolar. Informe técnico de investigación presentado a la División de Investigaciones de la Sede Bogotá-Universidad Nacional de Colombia (DIB-UN), el Instituto para el Desarrollo Pedagógico y la Innovación en Educación de la Alcaldía Mayor de Bogotá (IDEP) y Colciencias.

Flórez, R. y Moreno, M. (2006). Comunicación y lectura en el aula: un ecosistema. En Fundalectura (Colombia), Ministerio de CulturaBiblioteca Nacional (Colombia) y Embajada de Francia (Colombia) (org.), Memorias del Séptimo Congreso Nacional de Lectura y Cuarto Coloquio Colombofrancés de Bibliotecas (pp. 150-157). Bogotá: Organizadores.

Gopnik, A. (1999). Palabras, pensamientos y teorías. Madrid: Aprendizaje Visor.

Guido, S. (2006). Seminario Experiencias Pedagógicas, Políticas Educativas y Poblaciones Vulnerables 
(Apuntes). Programa académico de Maestría en Educación, Departamento de Posgrados, Facultad de Educación, Universidad Pedagógica Nacional, Bogotá, Colombia, segundo semestre.

Haken, H. (1999). Synergetics and some applications to psychology. En W. Tsacher y P. Daualder (ed.), Dynamics, synergetics, autonomous agents. Londres: World Scientific.

Hederich, C. (2007). Estilo cognitivo en la dimensión de dependencia-independencia de campo. Bogotá: Universidad Pedagógica Nacional.

Hederich, C., Camargo, A. y Reyes, M. (2005). Ritmos cognitivos en la escuela. Bogotá: Centro de Investigaciones de la Universidad Pedagógica Nacional, Ciup.

Hederich, C, y Camargo, A. (2002). Estilos cognitivos en Colombia. Bogotá: Colciencias-Centro de Investigaciones de la Universidad Pedagógica Nacional.

Jodelet, D. (1991). Representaciones sociales: fenómeno, conceptos y teoría. En S. Moscovici (ed.), Psicología social 1: conceptos básicos. Barcelona: Paidós.

Jörg, T. (2004). Complexity theory and the reinvention of reality of education. En B. Davies, R. Luce-Kapler y R. Upitis (ed.), Proceedings of the 2004 complexity science and educational research conferences (pp.121-146). University of Alberta: Institute for Complexity and Education.

Jörg, T. (2005). Complexity theory as a building stone for a new science of learning and education through peer interaction. Extraído desde http:// www.liv.ac.uk/ccr/2005_conf/index.htm

Karmiloff-Smith, A. (1992). Más allá de la modularidad. Madrid: Alianza Psicología.

Luria, A. (1998). Cerebro y lenguaje. Barcelona: Fontanella.

Mason, M. (2008). What is complexity theory and what are its implications for educational change? Educational Philosophy and Theory, 40(1), 35-49.

Maturana, H. y Varela, F. (1990). El árbol del conocimiento. Santiago de Chile: Editorial Universitaria.
Morin, E. (1982). Ciencia con conciencia. Barcelona: Anthropos.

Morin, E. (1996). Introducción al pensamiento complejo. Barcelona: Gedisa.

Orwell, G. (1961). Boy's Weeklies. En G. Orwell, Collected Essays (pp. 88-17). Londres: Secker y Warburg.

Piaget, J. (1971). El estructuralismo. Buenos Aires: Proteo.

Piaget, J. (1975). La equilibración de las estructuras cognoscitivas. Madrid: Siglo XXI Editores.

Piaget, J. (1976). Problemas de psicología genética. Barcelona: Ariel.

Puche-Navarro, R. (2003). La actividad mental del niño: una propuesta de estudio. En B. C. Orozco (comp.), El niño: científico, lector y escritor, matemático (pp. 17-40). Cali: Universidad del Valle, Centro de Investigaciones en Psicología, Cognición y Cultura.

Puche-Navarro, R. (2005). Los sistemas dinámicos en el estudio del desarrollo cognitivo. Conferencia dictada en el IV Encuentro de Investigadores en Ciencias del Comportamiento, Universidad Nacional de Colombia, Bogotá, Colombia.

Rogoff, B. (1995). Aprendices del pensamiento. Barcelona: Paidós.

Rumer, B. y Ryvkin, M. (1980). Thermodynamics, statistical physics, and kinetics. Moscú: MIR Publishers.

Saracho, O. (2003). Matching teachers' and students' cognitive styles. Early Child Development and Care, 173(2-3), 161-173.

Sperber, D. y Wilson, D. (1982). Relevance, communication and cognition. Oxford: Basil Blackwell.

Von Bertalanffy, L., Ashby, R. y Weinberg, G. (1978). Tendencias de la teoría general de sistemas. Madrid: Alianza.

Von Bertalanffy, L. (2000). Teoría general de los sistemas. Bogotá: Fondo de Cultura Económica.

Vygotski, L. (1979). El desarrollo de los procesos psicológicos superiores. Barcelona: Crítica.

Vygotski, L. (1995). Pensamiento y lenguaje. Barcelona: Paidós. 